PROCEEDINGS OF THE

AMERICAN MATHEMATICAL SOCIETY

Volume 127, Number 2, February 1999, Pages 315-321

S 0002-9939(99)05114-X

\title{
CLASSIFICATION OF EXTREMAL CONTRACTIONS FROM SMOOTH FOURFOLDS OF $(3,1)$-TYPE
}

\author{
HIROMICHI TAKAGI \\ (Communicated by Ron Donagi)
}

\begin{abstract}
We investigate divisorial contractions of extremal rays from smooth fourfolds. When the exceptional divisor is contracted to a curve, we prove that the divisor is a $\mathbb{P}^{2}$-bundle or quadric bundle over a smooth curve and the contraction is the blowing up along the curve. Furthermore we determine the local analytic structure of the contraction.
\end{abstract}

\section{INTRODUCTION}

Let $X$ be a projective normal variety with only terminal singularities and $Y$ a projective normal variety.

We say that $f: X \rightarrow Y$ is a contraction of an extremal ray if

(1) $f$ has only connected fibers;

(2) $-K_{X}$ is $f$-ample;

(3) for any two $f$-exceptional curves $C_{1}$ and $C_{2}$, there exists a positive rational number $r$ such that for any $\mathbb{Q}$-Cartier divisor $D,\left(D . C_{1}\right)=r\left(D . C_{2}\right)$.

We call the pull back of an ample divisor on $Y$ a good supporting divisor. Furthermore we say that such a contraction is of $(m, n)$-type if the dimension of the exceptional locus is equal to $m$ and the dimension of the image of the exceptional locus is equal to $n$. We also say that a contraction is divisorial if its exceptional locus is a divisor. (For a more general setting, see $[\mathrm{KMM}]$.)

When $\operatorname{dim} X=4$ and $X$ is smooth, many authors investigated contractions of extremal rays. Here are the references for them:

In $[\mathrm{A}], \mathrm{T}$. Ando determined the contractions of $(4,3)$-type and $(3,2)$-type in the case when all nontrivial fibers are 1-dimensional and also treated the contractions of (3,0)-type and (3,1)-type. (M. Beltrametti obtained similar results in [Be1].) In [A-W2], M. Andreatta and J. Wiśniewski determined isolated 2-dimensional fibers of the contractions of $(3,2)$-type. See also [A-W3] for the most recent developments in the study of higher dimensional contractions. In [Be2], M. Beltrametti listed the possibilities of the exceptional divisors of the contractions of $(3,0)$-type and in [F1], T. Fujita excluded many nonnormal possibilities in the list. In [Kac], Y. Kachi described isolated 2-dimensional fibers of the contraction of $(4,3)$-type. In [Kaw2], Y. Kawamata determined the contractions of (2,0)-type.

Received by the editors February 12, 1997 and, in revised form, April 24, 1997.

1991 Mathematics Subject Classification. Primary 14E30; Secondary 14J35.

Key words and phrases. Extremal ray, contraction morphism.

The author is a Research Fellow of the Japan Society for the Promotion of Science. 
In this paper we investigate the contractions of $(3,1)$-type.

Main Theorem. Let $X$ be a smooth 4-fold and let $f: X \rightarrow Y$ be a contraction of an extremal ray of $(3,1)$-type. Let $E$ be the exceptional divisor of $f$ and $C:=f(E)$. Then

(1) $C$ is a smooth curve;

(2) $\left.f\right|_{E}: E \rightarrow C$ is a $\mathbb{P}^{2}$-bundle or a quadric bundle (see Definition 1.4 below) over $C$ without multiple fibers;

(3) $f$ is the blowing up of $Y$ along $C$;

(4) (Local analytic description of singularity of $Y$ along $C$ )

(4p1) if $\left.f\right|_{E}: E \rightarrow C$ is a $\mathbb{P}^{2}$-bundle and $\left.\mathcal{O}_{X}(-E)\right|_{F} \simeq \mathcal{O}_{\mathbb{P}^{2}}(1)$ for any fiber $F$ of $\left.f\right|_{E}$, then $Y$ is smooth along $C$;

(4p2) if $\left.f\right|_{E}: E \rightarrow C$ is a $\mathbb{P}^{2}$-bundle and $\left.\mathcal{O}_{X}(-E)\right|_{F} \simeq \mathcal{O}_{\mathbb{P}^{2}}(2)$ for any fiber $F$ of $\left.f\right|_{E}$, then $(C \subset Y)$ is locally analytically isomorphic to $((x=y=z=0) \subset$ $\left.\left(\mathbb{C}^{3} / \mathbb{Z}_{2}(1,1,1)\right) \times \mathbb{C}\right)$, where $x, y, z$ are coordinates of $\mathbb{C}^{3}$ and $\mathbb{C}^{3} / \mathbb{Z}_{2}(1,1,1)$ means the quotient space of $\mathbb{C}^{3}$ by the action of $\mathbb{Z}_{2}$ which acts as $(x, y, z) \rightarrow$ $(-x,-y,-z)$;

(4q) if $\left.f\right|_{E}: E \rightarrow C$ is a quadric bundle, then $Y$ is analytically locally a hypersurface $f(x, y, z, w, t)=0$ in $\mathbb{C}^{5}$ with coordinates $x, y, z, w, t$ and $C$ is a local complete intersection $(x=y=z=w=0)$. Let $F^{\prime}$ be the fiber of $\left.f\right|_{E}$ over the origin and $F$ a general fiber near $F^{\prime}$;

(4q1) if $F$ and $F^{\prime}$ are $\mathbb{P}^{1} \times \mathbb{P}^{1}$, then we can write that

$$
f(x, y, z, w, t)=x^{2}+y^{2}+z^{2}+w^{2}
$$

(4q2) if $F$ is $\mathbb{P}^{1} \times \mathbb{P}^{1}$ and $F^{\prime}$ is $\mathbb{F}_{2,0}$, then we can write that

$$
f(x, y, z, w, t)=x^{2}+y^{2}+z^{2}+t^{m} w^{2}+w^{3} \text { for } m \geqq 2,
$$

or

$$
f(x, y, z, w, t)=x^{2}+y^{2}+z^{2}+t w^{2}+g(w, t) \text { with } g \in\left(w^{3}\right) ;
$$

(4q3) if $F$ is $\mathbb{P}^{1} \times \mathbb{P}^{1}$ and $F^{\prime}$ is the union of two distinct planes in $\mathbb{P}^{3}$, then we can write that

$$
\begin{array}{r}
f(x, y, z, w, t)=x^{2}+y^{2}+t^{m} z^{2}+z^{3}+t w^{2}+g(t, z, w) \\
\text { with } m \geqq 2 \text { and } g \in\left(z^{2} w, z w^{2}, w^{3}\right),
\end{array}
$$

or

$$
\begin{array}{r}
f(x, y, z, w, t)=x^{2}+y^{2}+t z^{2}+t w^{2}+g(t, z, w) \text { with } g \in(z, w)^{3} \text { and } \\
g_{3}(z, w) \text { cannot be divided by } z+\sqrt{-1} w \text { or } z-\sqrt{-1} w \\
\text { where } g_{3} \text { is the homogeneous part of } g \text { of degree } 3 \text { in } z, w ;
\end{array}
$$

(4q4) if $F$ and $F^{\prime}$ are $\mathbb{F}_{2,0}$, then we can write that

$$
f(x, y, z, w, t)=x^{2}+y^{2}+z^{2}+w^{3} ;
$$

(4q5) if $F$ is $\mathbb{F}_{2,0}$ and $F^{\prime}$ is the union of two planes in $\mathbb{P}^{3}$, then we can write that

$$
f(x, y, z, w, t)=x^{2}+y^{2}+t z^{2}+w^{3}+g(t, z, w) \text { with } g(t, z, w) \in\left(z^{3}, z^{2} w, z w^{2}\right) .
$$

Notation and conventions. (1) In this paper, we will work over $\mathbb{C}$, the complex number field. 
(2) The $\mathbb{P}^{1}$-bundle $\mathbb{P}\left(\mathcal{O}_{\mathbb{P}^{1}} \oplus \mathcal{O}_{\mathbb{P}^{1}}(-a)\right)$ over $\mathbb{P}^{1}$, a Hirzebruch surface of degree $a$, is denoted by $\mathbb{F}_{a}$. Its unique negative section is denoted by $C_{0}$ and a ruling is denoted by $f$. The projective cone obtained from $\mathbb{F}_{a}$ by the contraction of $C_{0}$ is denoted by $\mathbb{F}_{a, 0}$. A generating line on $\mathbb{F}_{a, 0}$ is denoted by $l$.

\section{Preliminaries}

Theorem 1.1. Let $X, Y, E$ and $C$ be as in the main theorem. Let $F$ be a general fiber of $\left.f\right|_{E}: E \rightarrow C$. Then $\left(F,-\left.K_{X}\right|_{F}\right) \simeq\left(\mathbb{P}^{2}, \mathcal{O}_{\mathbb{P}^{2}}(1)\right),\left(\mathbb{P}^{2}, \mathcal{O}_{\mathbb{P}^{2}}(2)\right)$, $\left(\mathbb{P}^{1} \times \mathbb{P}^{1}, \mathcal{O}_{\mathbb{P}^{1}}(1) \otimes \mathcal{O}_{\mathbb{P}^{1}}(1)\right)$ or $\left(\mathbb{F}_{2,0},\left.\mathcal{O}_{\mathbb{P}^{3}}(1)\right|_{\mathbb{F}_{2,0}}\right)$.

Proof. In [A, Theorem 2.1], T. Ando stated this theorem but his proof of irreducibility of $F$ is insufficient. Since the image of $E$ by $\left.H\right|_{E}$, where $H$ is a good supporting divisor, is 1-dimensional, Bertini's theorem does not derive the irreducibility of $F$. Since we don't know that $E$ is normal a priori, irreducibility of $F$ is subtle. So we will prove it here. Once we prove it, the results follow from [A, Theorem 2.1]. (M. Beltrametti stated a slightly weaker result in [Be1, Proposition 2.7].) We assume that $F$ is reducible and obtain a contradiction. Let $H$ be a good supporting divisor of $f$. We may assume that $H$ is a smooth variety and locally $F=H \cap E$. By the adjunction formula, $-K_{F}=-K_{X}-E-\left.H\right|_{F}$, but since $\left.H\right|_{F} \sim 0$, we have $-K_{F}=-K_{X}-\left.E\right|_{F}$. Note that $-\left.K_{X}\right|_{F}$ and $-\left.E\right|_{F}$ are ample Cartier divisors on $F$. In particular $-K_{F}$ is ample on $F$ and hence $F$ is a generalized del Pezzo surface (i.e., a Gorenstein (possibly reducible) anticanonically polarized surface). Write $F=\bigcup F_{i}$, where $F_{i}$ is an irreducible component of $F$. By [R3, 1.1], $\left(F_{i},-\left.K_{F}\right|_{F_{i}}\right)$ is one of the following:

(a) $\left(\mathbb{P}^{2}, \mathcal{O}_{\mathbb{P}^{2}}(i)\right)$, where $i$ is 1 or 2 ;

(b) $\left(\mathbb{F}_{a, 0}, \mathcal{O}_{\mathbb{F}_{a, 0}}(a l)\right)$;

(c) $\left(\mathbb{F}_{a}, \mathcal{O}_{\mathbb{F}_{a}}\left(C_{0}+(a+1) f\right)\right)$;

(d) $\left(\mathbb{F}_{a}, \mathcal{O}_{\mathbb{F}_{a}}\left(C_{0}+(a+2) f\right)\right)$.

But since $-K_{F}$ is a sum of two ample Cartier divisors, (a) with $i=1$, (b), (c) and (d) are impossible. So we obtain that $\left(F_{i},-\left.K_{F}\right|_{F_{i}}\right) \simeq\left(\mathbb{P}^{2}, \mathcal{O}_{\mathbb{P}^{2}}(2)\right)$ and $-\left.K_{X}\right|_{F_{i}} \simeq$ $\mathcal{O}_{\mathbb{P}^{2}}$ (1). Furthermore by [R3, 1.3, 1.4 and 1.5], $F$ is a union of two $\mathbb{P}^{2}$ 's which intersect along a line on them. But this is impossible since $N_{F_{i} / H} \simeq \mathcal{O}_{\mathbb{P}^{2}}(-2)$ and hence there is a bimeromorphic contraction which contracts only one $F_{i}$.

The next theorem about freeness is very useful for the classification of low dimensional fibers of an extremal contraction.

Theorem 1.2 (M. Andreatta and J. Wiśniewski). Let $X$ and $Y$ be normal log terminal varieties and $f: X \rightarrow Y$ a projective morphism. Let $L$ be an $f$-ample line bundle on $X$ and $F$ a fiber of $f$. Assume that $f$ is the adjoint contraction supported by $K_{X}+r L$ and either $\operatorname{dim} F<r+1$ if $\operatorname{dim} Y<\operatorname{dim} X$ or $\operatorname{dim} F \leq r+1$ if $\operatorname{dim} Y=\operatorname{dim} X$.

Then $f^{*} f_{*} L \rightarrow L$ is surjective at every point of $F$, i.e., $L$ is $f$-free at every point of $F$.

Proof. See [A-W1, Theorem 3.1]. Andreatta and Wiśniewski assume that $L$ is ample but their proof works also for the case that $L$ is relatively ample.

Theorem 1.3 (P. M. H. Wilson). Let $X_{0}$ be a smooth 3-fold and $Y_{0}$ a 3-fold with only canonical singularities. Let $f: X_{0} \rightarrow Y_{0}$ be a crepant birational (i.e., $f$ satisfies $K_{X_{0}}=f^{*} K_{Y_{0}}$ ) projective morphism which contracts an irreducible divisor 
to a curve. Let $E_{0}$ be the exceptional divisor and $C:=f\left(E_{0}\right)$. Assume that $-E_{0}$ is $f$-ample. Then $C$ is a smooth curve and $Y_{0}$ has only $c D V$ singularity along $C$.

Proof. See [W1, Theorem 2.2] and [W2, Proposition 3.1]. Wilson assumes that $X_{0}$ is a Calabi-Yau manifold and $f$ is primitive. But by the following modification, his proof works in the local situation and under the assumption that $-E_{0}$ is $f$-ample instead of the assumption that $f$ is primitive. In Wilson's proof [W1, the 6 th line of page 569], he claimed that $f$ coincides with the blowing up of $Y_{0}$ along $C$ using primitivity of $f$. We replace the argument as follows:

Let $f^{\prime}: X_{0}^{\prime} \rightarrow Y_{0}$ be the blowing up of $Y_{0}$ along $C$ and $E_{0}^{\prime}$ the exceptional divisor. By the universal property of blowing up, $g: X_{0} \rightarrow X_{0}^{\prime}$ is naturally defined. We know that $g\left(E_{0}\right)=E_{0}^{\prime}$ since $E_{0}$ is irreducible. Note that $E_{0}^{\prime}$ is a Cartier divisor by the property of blowing up. If $g$ is not an isomorphism, it contracts finitely many curves since $E_{0}$ is irreducible. But it is impossible since $E_{0}^{\prime}$ is Cartier and $-E_{0}$ is positive for the contracted curves by the assumption that $-E_{0}$ is $f$-ample.

Definition 1.4. Let $E$ be a normal projective 3-fold and $C$ a smooth curve. Let $f: E \rightarrow C$ be a projective surjective morphism. We say that $f: E \rightarrow C$ is a quadric bundle if the following conditions are satisfied:

(1) There exists a $f$-very ample line bundle $L$ on $E$ and $f_{*} L$ is locally free of rank 4

(2) $L$ gives the embedding $E \rightarrow \mathbb{P}\left(f_{*} L\right)$ over $C$;

(3) For any closed point $s$ of $C, E_{s}$ (the fiber of $f$ over $s$ ) is a quadric in $\mathbb{P}^{3} \simeq$ $\mathbb{P}\left(f_{*} L\right)_{s}$ (the fiber of $\mathbb{P}\left(f_{*} L\right) \rightarrow C$ over $s$ ).

If we can take such an $L$, we say that $f: E \rightarrow C$ is the quadric bundle associated to $L$.

Remark 1.5. We require that $E$ is normal in Definition 1.4 so general fibers of $f$ are irreducible.

\section{Proof of the MAIN THEOREM}

Proof of (1). Since $L:=-K_{X}$ is $f$-ample, we can apply Theorem 1.2 for this $f$ and $L$ with $r=1$. (Remark that the dimension of fibers of $f$ is 2.) Then for any point $P$ of $C$ and a suitable affine neighborhood $U$ of $P$ in $Y,|L|_{f^{-1}(U)} \mid$ has no base points on $f^{-1}(U)$. Let $X_{0}$ be a general smooth member of $|L|_{f^{-1}(U)} \mid, Y_{0}:=f\left(X_{0}\right)$ and $E_{0}:=\left.E\right|_{X_{0}}$. Then $\left.f\right|_{X_{0}}$ is crepant, $E_{0}$ is irreducible (by Bertini's theorem) and $-E_{0}$ is $\left.f\right|_{X_{0}}$-ample. From this, we can apply Theorem 1.3 for $X_{0}, Y_{0}$ and $E_{0}$. Hence $C$ is smooth.

Proof of (2). Let $F$ be a general fiber of $f$.

If $\left(F,-\left.K_{X}\right|_{F}\right) \simeq\left(\mathbb{P}^{2}, \mathcal{O}_{\mathbb{P}^{2}}(1)\right)$, let $L^{\prime}$ be $\mathcal{O}_{E}\left(-K_{X}\right)$.

If $\left(F,-\left.K_{X}\right|_{F}\right) \simeq\left(\mathbb{P}^{2}, \mathcal{O}_{\mathbb{P}^{2}}(2)\right),\left(\mathbb{P}^{1} \times \mathbb{P}^{1}, \mathcal{O}_{\mathbb{P}^{1}}(1) \otimes \mathcal{O}_{\mathbb{P}^{1}}(1)\right)$ or $\left(\mathbb{F}_{2,0},\left.\mathcal{O}_{\mathbb{P}^{3}}(1)\right|_{\mathbb{F}_{2,0}}\right)$, let $L^{\prime}$ be $\mathcal{O}_{E}(-E)$. Then we will prove that

(i) if $F \simeq \mathbb{P}^{2},\left.f\right|_{E}: E \rightarrow C$ is a $\mathbb{P}^{2}$-bundle $\mathbb{P}\left(\left(\left.f\right|_{E}\right)_{*} L^{\prime}\right)$;

(ii) if $F \simeq \mathbb{P}^{1} \times \mathbb{P}^{1}$ or $\mathbb{F}_{2,0},\left.f\right|_{E}: E \rightarrow C$ is the quadric bundle associated to $L^{\prime}$.

Claim 2.1. $\left(\left.f\right|_{E}\right)_{*} L^{\prime}$ is locally free.

Proof. By the proof of (1) above, $C$ is smooth so $\left.f\right|_{E}$ is flat. Consider the exact sequence

$$
0 \rightarrow \mathcal{O}_{X}\left(-K_{X}-E\right) \rightarrow \mathcal{O}_{X}\left(-K_{X}\right) \rightarrow L^{\prime} \rightarrow 0
$$


if $L^{\prime}=\mathcal{O}_{E}\left(-K_{X}\right)$ or

$$
0 \rightarrow \mathcal{O}_{X}(-2 E) \rightarrow \mathcal{O}_{X}(-E) \rightarrow L^{\prime} \rightarrow 0
$$

if $L^{\prime}=\mathcal{O}_{E}(-E)$. By the Kodaira-Kawamata-Viehweg vanishing theorem, we have $R^{i}\left(\left.f\right|_{E}\right)_{*} L^{\prime}=0$ for any $i>0$. On the other hand, we have $H^{i}\left(E_{s}, L_{s}^{\prime}\right)=0$ for $i \gg 0$ and any $s \in C$. In particular $L^{\prime}$ is flat over $C, R^{i}\left(\left.f\right|_{E}\right)_{*} L^{\prime} \otimes k(s)=H^{i}\left(E_{s}, L_{s}^{\prime}\right)$ for $i \gg 0$ and $R^{i}\left(\left.f\right|_{E}\right)_{*} L^{\prime}$ is locally free for any $i>0$. So by using the cohomology and base change theorem inductively (cf. [H, III Theorem 12.11]), we know that $\left(\left.f\right|_{E}\right)_{*} L^{\prime}$ is locally free.

Claim 2.2. $L^{\prime}$ is $\left.f\right|_{E}$-free.

Proof. We consider the commutative diagram

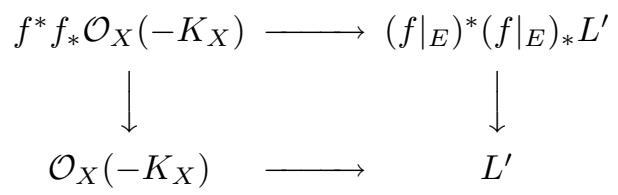

if $L^{\prime}=\mathcal{O}_{E}\left(-K_{X}\right)$ or

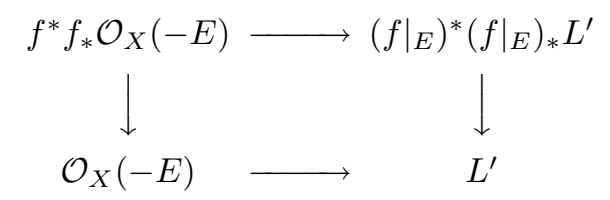

if $L^{\prime}=\mathcal{O}_{E}(-E)$.

We see the left vertical arrow is surjective by Theorem 1.2 and so is the bottom arrow clearly. So the right vertical arrow must be surjective, i.e., $L^{\prime}$ is $\left.f\right|_{E}$-free.

By these, we obtain the morphism $g: E \rightarrow \mathbb{P}\left(\left(\left.f\right|_{E}\right)_{*} L^{\prime}\right)$ defined by $L^{\prime}$.

If we are in case (i), $g$ is birational since the general fiber of $f$ is $\mathbb{P}^{2}$ and $\mathbb{P}\left(\left(\left.f\right|_{E}\right)_{*} L^{\prime}\right)$ is a $\mathbb{P}^{2}$-bundle. Furthermore $g$ is finite since $L^{\prime}$ is $\left.f\right|_{E}$-ample. So by Zariski's Main Theorem, $g$ is an isomorphism and we are done.

If we are in case (ii), we will show first that $f$ is the blowing up of $Y$ along $C$.

Let $f^{\prime}: X^{\prime} \rightarrow Y$ be the blowing up of $Y$ along $C$. By Theorem 1.2, we have the surjection $f^{*} f_{*} \mathcal{O}_{X}(-E) \rightarrow \mathcal{O}_{X}(-E)$. Since $f_{*} \mathcal{O}_{X}(-E)=\mathcal{I}_{C}$, where $\mathcal{I}_{C}$ is the ideal sheaf of $C$, we have the morphism $h: X \rightarrow X^{\prime}$ which satisfies $f=f^{\prime} \circ h$ by the universal property of blowing up. By Theorem 1.3 and the proof of the main theorem (1), near any point $x$ on $C$, there is a general member of $\left|-K_{Y}\right|$ through $x$ which has only cDV singularities along $C$. Since $\left|-K_{Y}\right|$ is a Cartier divisor in case (ii), $Y$ also has hypersurface singularities along $C$. If we consider $f^{\prime}$ analytically locally near $x$, we know $X^{\prime}$ also has hypersurface singularities since $Y$ is a hypersurface in $\mathbb{C}^{5}$ and $C$ is a smooth curve. Furthermore $X^{\prime}$ is nonsingular along a general fiber of $f^{\prime}$ since $f$ and $f^{\prime}$ coincide over a general point of $C$. Hence $X^{\prime}$ is normal. Since $f$ contracts only an irreducible divisor, $h$ contracts no divisor. If $h$ is not finite, $h$ is a small contraction such that for any contracted curve $m$, $-K_{X} \cdot m<0$. But because $X^{\prime}$ is Gorenstein, this is impossible. Hence $h$ is finite birational, which in turn shows $h$ is an isomorphism by Zariski's Main Theorem.

By this, $E$ is naturally embedded in $\mathbb{P}\left(\left(\left.f\right|_{E}\right)_{*} L^{\prime}\right)$. This embedding coincides with $g$. We can easily check that $E$ is the quadric bundle associated to $L^{\prime}$

We complete the proof of the main theorem (2) without excluding multiple fibers in case (ii). This is done in the proof of the main theorem (4). 
Proof of (3). In case $E$ is a quadric bundle, the proof was given in the proof of (2). In case $E$ is a $\mathbb{P}^{2}$-bundle and $\left(F,-\left.E\right|_{F}\right) \simeq\left(\mathbb{P}^{2}, \mathcal{O}_{\mathbb{P}^{2}}(1)\right)$ for a fiber $F$ of $f$, this is well known (see $[\mathrm{N}])$. In case $E$ is a $\mathbb{P}^{2}$-bundle and $\left(F,-\left.E\right|_{F}\right) \simeq\left(\mathbb{P}^{2}, \mathcal{O}_{\mathbb{P}^{2}}(2)\right)$ for a fiber $F$ of $f$, we can proceed as follows: let $P$ be any point of $C$ and take a general hyperplane section $A$ through $P$. Let $H$ be the pull back of $A$. Then $H$ is smooth along $\left.E\right|_{H} \simeq \mathbb{P}^{2}$ since $\left.E\right|_{H}$ is smooth and a Cartier divisor of $H$. So $\left.f\right|_{H}$ is a contraction of an extremal ray from a smooth 3-fold near the fiber over $P$. Then by Mori's classification [Mo2], $A$ has a singularity analytically isomorphic to $o \in \mathbb{C}^{3} / \mathbb{Z}_{2}(1,1,1)$ at $P$ and $\left.f\right|_{H}$ is the blowing up of $A$ at $P$. Hence we are done.

Proof of (4). (4p1) This is well known (see $[\mathrm{N}])$.

$(4 \mathrm{p} 2) o \in \mathbb{C}^{3} / \mathbb{Z}_{2}(1,1,1)$ is rigid by $[\mathrm{S}]$, so we are done.

(4q) By the proof of (2), $Y$ is locally a hypersurface in $\mathbb{C}^{5}$ and $C$ is a local complete intersection in $\mathbb{C}^{5}$. The rest are easy calculations, so we treat only the following for a sample.

Claim 2.5. $F^{\prime}$ is not multiple.

Proof. Assume that $F^{\prime}$ is multiple. We treat only the case $F$ is $\mathbb{P}^{1} \times \mathbb{P}^{1}$. By the proof of (2), we can assume that $f(x, y, z, w, t)=x^{2}+t^{m} y^{2}+t^{n} z^{2}+t^{l} w^{2}+g(y, z, w, t)$ with $g(y, z, w, t) \in(y, z, w)^{3}$. We will show that the 4 -fold obtained by the blow up must be singular (we say in short that the blow up is singular). For simplicity, we assume furthermore that $m=n=l=1$. By easy computations, the blow up is singular if and only if $x=t=y^{2}+z^{2}+w^{2}=g_{3}(y, z, w, t)=0$ has a solution. But the equation always has a solution, so we are done.

This completes the proof of the main theorem.

\section{ACKNOWLEDGMENT}

I am very thankful to Professor Yujiro Kawamata for arousing my interest in this object and encouraging me during the preparation of this paper. I am grateful to Professor Takao Fujita for giving me useful advice. I am thankful to Dr. Yoshiaki Fukuma for giving me much advice. I am grateful to Dr. Hironobu Ishihara for pointing out many English mistakes and giving me $\mathrm{T}_{\mathrm{EX}} \mathrm{X}$ advice. I express my hearty thanks to Dr. Nobuyoshi Takahashi for pointing out a mistake in the first draft of this paper.

\section{REFERENCES}

[A] T. Ando, On extremal rays of the higher dimensional varieties, Invent. Math. 81 (1985), 347-357. MR 87g:14045

[A-W1] M. Andreatta and J. Wiśniewski, A note on nonvanishing and applications, Duke Math. J. 72 (1993), 739-755. MR 95c:14007

[A-W2] _ On contractions of smooth varieties, J. Algebraic Geom. 7 (1998), 253-312. CMP 98:12

[A-W3] _ A view on contractions of higher dimensional varieties, Proc. Sympos. Pure Math., vol. 62, Amer. Math. Soc., Providence, RI, 1997, pp. 153-183. CMP 98:07

[Be1] M. Beltrametti, On d-folds whose canonical bundle is not numerically effective,According to Mori and Kawamata, Ann. Mat. Pura. Appl. 147 (1987), 151-172. MR 89c:14063

[Be2] Contraction of non numerically effective extremal rays in dimension 4 , TeubnerTexte Math. 92 (1986), 24-37. MR 89c:14064

[F0] T. Fujita, Classification theories of polarized varieties, London Math. Soc. Lecture Note Ser. 155 (1990). MR 93e:14009 
[F1] — On singular del Pezzo varieties, Lecture Notes in Math., vol. 1417, SpringerVerlag, 1990, p. 117-128. MR 91b:14057

[H] R. Hartshorne, Algebraic Geometry, GTM 52 (1977). MR 57:3116

[Kac] Y. Kachi, Extremal contractions from 4-dimensional manifolds to 3-folds, Ann. di Pisa (4) 24 (1997), 63-131. CMP 98:03

[Kaw1] Y. Kawamata, The cone of curves of algebraic varieties, Ann. of Math. 119 (1984), 603-633. MR 86c:14013b

[Kaw2] - Small contractions of four dimensional algebraic manifolds, Math. Ann. 284 (1989), 595-600. MR 91e:14039

[KMM] Y. Kawamata, K. Matsuda and K. Matsuki, Introduction to the minimal model problem, Adv. St. Pure Math. 10 (1987), 287-360. MR 89e:14015

[Mo1] S. Mori, Projective manifolds with ample tangent bundles, Ann. of Math. 110 (1979), 593-606. MR 81j:14010

[Mo2] , Threefolds whose canonical bundles are not numerically effective, Ann. of Math. 116 (1982), 133-176. MR 84e:14032

[N] S. Nakano, On the inverse of monoidal transformations, Publ. RIMS Kyoto Univ. 6 (1971), 483-502. MR 45:3778; MR 45:3779; MR 45:3780

[R1] M.Reid, Canonical 3-folds, Journées de Géométrie Algébrique d'Angers, Sijthoff and Noordhoff, Alphen, 1980, p. 273-310. MR 82i:14025

[R2] Minimal models of canonical 3-folds, Adv. St. Pure Math. 1 (1983), 131-180. MR 86a: 14010

[R3] , Nonnormal del Pezzo surfaces, Publ. RIMS Kyoto Univ. 30 (1994), 695-727. MR 96a:14042

[S] M.Schlessinger, Rigidity of quotient singularities, Invent.Math. 14 (1971), 17-26. MR 45:1912

[W1] P. M. H. Wilson, The Kähler cone on Calabi Yau threefolds (and Erratum), Invent. Math. 107 (114) (1992 (1993)), 561-583 (231-233). MR 93a:14037; MR 94i: 14042

[W2] , Symplectic Deformations of Calabi-Yau threefolds, J. Diff. Geom. 45 (1997), 611-637. CMP 98:02

Department of Mathematical Sciences, University of Tokyo, Komaba, Meguro-ku, TOKYO 153-0041, JAPAN

E-mail address: htakagi@ms.u-tokyo.ac.jp 